\title{
Mac-kompatible Praxissoftware
}

\author{
Viele Praxen würden ihre Abläufe gerne mit Apple-Rechnern ver- \\ walten. Das Angebot an Mac-kompatiblen Praxissoftware-Paketen \\ ist zwar klein, aber fein.
}

So mancher Mediziner schätzt die Geräte von Apple. Dabei stellt sich allerdings die Frage, ob es empfehlenswerte Praxissoftware-Lösungen für das Apple-Betriebssystem Mac OS X gibt. Im Vergleich zu den weit über 100 angebotenen Paketen für Windows ist die Auswahl hier deutlich kleiner - was aber nicht bedeutet, dass sie qualitativ schlechter wäre.

Gemäß der jüngsten verfügbaren Statistik der KBV wurde die Software „Medys" Ende 2014 in insgesamt 915 deutschen Praxen eingesetzt und kann damit auf die meisten Installationen Mac-kompatibler Praxissoftware-Pakete verweisen. Das Programm wird jedoch sowohl für Mac OS X als auch für Windows angeboten, und die exakte Zahl der Installationen pro Betriebssystem wird weder von der KBV ausgewiesen noch vom Hersteller veröffentlicht. Wie es für plattformübergreifende Lösungen typisch ist, bietet Medys nicht zu hundert Prozent den Mac-typischen "Look and Feel“ das System hat aber den Vorteil, dass es bei Bedarf auch in gemischten Umgebungen aus Mac- und Windows-PC eingesetzt werden kann.

\section{Mediziner als Ideengeber}

Der in Wülfrath ansässige Anbieter blickt auf eine 20-jährige Entwicklungsgeschichte seiner Software zurück, an der von Anfang an auch Mediziner beteiligt waren. Das Programm erlaubt es, beliebige Dokumente per „Drag and Drop“ einer Patientenakte zuzuordnen. Für die Erstellung von Arztbriefen ist eine vollwertige Textverarbeitung integriert. Zudem enthält das Paket ein umfangreiches Sortiment an BG- und KV-Formularen. Ein spezielles Hausbesuchsmodul erlaubt die Übertragung einzelner Patientenakten aus dem Praxissystem auf einen Laptop.

Etwas über 300 Installationen erreicht das Programm "Tomedo“ vom Jenaer Softwarehaus Zollsoft. Das Unternehmen bietet seine Lösung seit Ende 2013 an. Die beiden Firmengründer sind Brü- der und stammen aus einer Arztfamilie - ihre Eltern waren die ersten Anwender und Tester des Programms. Neben einem Grundpaket mit den üblichen Standardfunktionen wie Patientenverwaltung und KV-, BG- sowie Privatabrechnung bietet die Software Module, die je nach Praxis beziehungsweise Klinik hinzu kombiniert werden können: etwa eine OP- und Bettenplanung, Labordatenübertragung oder Anbindung von Diagnostikgeräten.

\section{Extra Mobilfunktionen \\ für das iPad}

Neben der Unterstützung von Mac-PC bietet „Tomedo“ auch spezielle Funktionen für Apples Mobilgeräte: So können Ärzte auf Hausbesuchen entweder ein Mac-Notebook oder auch ein iPad mitnehmen und damit auf die Daten der Patienten zugreifen. Die Tablet-Nutzung hilft beispielsweise auch bei Visiten, um den Patienten Diagnosen oder Heilungsverlauf zu vermitteln. Mit der Kamera eines iPhone oder iPad lassen sich zum Beispiel Verletzungen oder Hautveränderungen fotografieren und direkt in die digitale Krankenakte eintragen.

Mit der in Apple-Geräten eingebauten Spracherkennung können Mediziner Diagnosen oder Notizen diktieren - die Software wandelt sie in lesbaren Text um und trägt diesen in der Krankenakte ein. Ein weiteres Modul erlaubt die digitale Erfassung von Erst-Anamnesen direkt auf dem iPad.

\section{Selbst für Linux-Systeme nutzbar}

Mit rund 30 Installationen laut KBV-Statistik zählt die Münchner Arkandus $\mathrm{GmbH}$ zu den kleineren Anbietern von Mac-tauglicher Praxissoftware. „Arkandus" wurde 2003 im Rahmen eines Projektauftrags entwickelt und seither zum Praxismanagementsystem für kassenärztliche Praxen, Kliniken und MVZ weiterentwickelt. Wie Medys ist die Software wahlweise auf Windows oder Mac OS X erhältlich - zusätzlich bieten die
Oft läuft Mac-Software - zum Teil in abgespeckter Form - auch direkt auf iPad oder iPhone.

Münchner auch eine Version für das Betriebssystem Linux an. Diese Flexibilität ist möglich, weil der Kern der Software in der plattformunabhängigen Programmsprache Java entwickelt wurde. Im Zentrum steht die Patienten- und Praxisverwaltung, die auch in diesem Fall Funktionen wie digitale Patientenakten, Briefschreibung, KV- und Privatabrechnung und die Anbindung an bildgebende Diagnostikgeräte bietet. Als Besonderheit lassen sich auch Module für Controlling und Qualitätsmanagement integrieren. Seit 2012 ist diese Software ebenfalls von der KBV zur kassenärztlichen Abrechnung zugelassen.

\section{Flexible Preisgefüge}

Die Preise der aufgeführten Programme möchte übrigens keiner der Hersteller pauschal nennen - sie hängen vom gewünschten Funktionsumfang ab, häufig stehen unterschiedliche Miet- oder Finanzierungsmodelle zur Wahl. Kleinere Praxen sollten den Einsatz einer Mackompatiblen Praxissoftware mit monatlichen Kosten ab etwa $150 €$ veranschlagen. Zusätzliche Funktionsmodule kosten extra. Ein Basisangebot zur telefonischen Unterstützung ist in den laufenden Gebühren üblicherweise enthalten, umfangreiche Schulungen, aufwändigere Anpassungen oder individuelle Problemlösungen werden von den Softwarehäusern oder ihren Vertriebspartnern hingegen extra abgerechnet. Entsprechende Angebote unterbreiten alle genannten Anbieter auf Anfrage. Oft übernimmt dann auch ein auf Kunden aus dem medizinischen Bereich spezialisiertes Systemhaus die Betreuung, sodass Ärzte die Lizenzkosten der Software und die benötigte Hardware als Gesamtpaket leasen oder finanzieren können.

Hannes Rügheimer 